\title{
3 Research Square \\ Clinical research of lower extremity exoskeleton robot In post-stroke hemiplegic patients
}

\section{Kan Wang ( $\nabla$ wangkan0526@sjtu.edu.cn )}

Shanghai Jiao Tong University School of Medicine Affiliated Renji Hospital https://orcid.org/00000001-9811-4142

\section{Haojun Yu}

Shanghai Jiao Tong University School of Medicine Affiliated Renji Hospital

\section{Huimin Zhong}

Shanghai Jiao Tong University Medical School Affiliated Ruijin Hospital

\section{Jing Peng}

Shanghai Jiao Tong University School of Medicine Affiliated Renji Hospital

\section{Weixin Yan}

shanghai jiaotong university school of mechanical engineering

\section{Ping Huang}

Shanghai Jiao Tong University Medical School Affiliated Ruijin Hospital

\section{Yangtai Guan}

Shanghai Jiao Tong University School of Medicine Affiliated Renji Hospital

\section{Research}

Keywords: stroke, exoskeleton robot, hemiplegia, gait, rehabilitation

Posted Date: June 19th, 2020

DOI: https://doi.org/10.21203/rs.3.rs-36484/v1

License: (c) (1) This work is licensed under a Creative Commons Attribution 4.0 International License. Read Full License 


\section{Abstract}

Background In recent years, the number of people suffering from stroke-induced motor function lost increased considerably. Recovery of motility is essential in improving their quality of life. However, the existing rehabilitation methods cannot fulfill patients' training requirements. As a new rehabilitation technology, exoskeleton robot provides a new treatment scheme for post-stroke hemiplegic patients. Objective To explore the safety and effectiveness of exoskeleton-assisted gait training for post-stroke hemiplegic patients. Methods A lower extremity hip joint exoskeleton robot was designed by the cooperation team, and 9 post-stroke hemiplegic patients were included for exoskeleton-assisted gait training. The three-dimensional gait parameters, plantar pressure and surface electromyography were used to validate the effectiveness of the exoskeleton robot in post-stroke hemiplegic patients. Results Exoskeleton-assisted ambulation training for post-stroke hemiplegic patients can correct the asymmetry of gait and abnormal phase of gait cycle, increase the toe-ground clearance and the angle of hip joint, reduce the pressure of non-paretic plantar and the impulse of bilateral feet, which help balance the plantar pressure distribution. It can also improve the integral electromyography value of specific muscle, which means that corresponding muscles are stimulated to generate activities. Conclusions The exoskeleton robot designed by our team can correct the hemiplegic gait and foot drop phenomenon of post-stroke patients, protecting them from damage caused by long-term abnormal gait, which contributes to the recovery of lower extremity motor function in hemiplegic patients. The results also provide important information for the design of lower extremity exoskeleton robot. In the future, we should further explore the rehabilitation function of exoskeleton robot in post-stroke hemiplegic patients, as well as the ambulation and rehabilitation function in other lower extremity motor dysfunction patients.

\section{Introduction}

Stroke is a medical problem facing the whole world, which is characterized by high morbidity, recurrence, mortality and disability ${ }^{[1]}$. Moreover, there are very limited treatment methods for stroke and the only drug approved by the FDA (Food and Drug Administration, USA) is tPA (tissue plasminogen activator) ${ }^{[2]}$. A study conducted by American Heart Association including millions of cerebral infarction patients shows that only $3.7 \%$ of them used tPA to thrombolysis ${ }^{[3]}$. Up to $80 \%$ stroke patients are leaved with varying degrees of disabilities due to the limited treatment methods ${ }^{[4]}$. The disability of motor function, especially the malperformance of lower limb, seriously affects their quality of life, so it is important to reestablish their motor function safely and effectively ${ }^{[5]}$. The rehabilitation guidelines and clinical experience have proved that early and scientific rehabilitation training can reduce the disability rate of stroke and improve the quality of patients' life ${ }^{[6]}$. At present, the rehabilitation of patients' lower extremity motor function mostly depends on therapists for one-to-one training, which can hardly meet the requirement of highintensity and personalized training, and the curative effect is difficult to be objectively evaluated ${ }^{[4]}$.

Exoskeleton robot, as a burgeoning rehabilitation technology, has been greatly developed in recent years $^{[7]}$. Exoskeleton robot is a kind of wearable bionic device, which integrates rehabilitation medicine, 
robotics and artificial intelligence ${ }^{[8]}$. Its application in the field of rehabilitation medicine is a research hotspot in various countries ${ }^{[9]}$. Compared with traditional rehabilitation methods, it can not only provide highly controllable, high-intensity and personalized rehabilitation training to relieve clinical staff of huge burden, but also can evaluate the improvement of motor function objectively and accurately ${ }^{[10]}$. Latest research results in the field of neural rehabilitation indicate that the central nervous system is highly plastic, which provides theoretical basis for the combination of rehabilitation medicine and robot technology, thus driving the application of exoskeleton robot in stroke rehabilitation to the focus direction ${ }^{[11,12]}$.

By the end of January 1, 2020, searching the keywords "stroke" and "exoskeleton" in the U.S. clinical trial database turns out 70 clinical studies, among them 26 are related to lower extremity motor dysfunction. Through the analysis of the above clinical researches, it is found that the rehabilitation effect of exoskeleton robot varies in different stages of stroke and different joints of limbs ${ }^{[13-16]}$, the key to improving its clinical effect lies in the mechanism design and software control ${ }^{[17-20]}$, and there is no mature research paradigm of gait training assisted by exoskeleton robot for stroke patients ${ }^{[21-23]}$.

Stroke patients display special hemiplegic gait and foot drop phenomenon ${ }^{[24]}$, which we aims to correct in this study by designing a hip joint exoskeleton, also with the hope of improving prognosis. To evaluate the curative effect objectively, comprehensively and accurately, we used three-dimensional motion capture system, conducted surface electromyogram (sEMG) and recorded plantar pressure.

\section{Materials And Methods}

\subsection{Research protocol}

1) Objective: To explore the safety and effectiveness of exoskeleton-assisted gait training for post-stroke hemiplegic patients.

2) Study design: prospective, non-randomized, self-controlled clinical study.

3) Sample size: about 10 post-stroke hemiplegic patients.

4) Inclusion criteria:
a. Age > 18 years;
b. Anterior circulation ischemic stroke attacked one month to two years before admission;
c. Unilateral limb dyskinesia (paretic side muscle strength level is III or above);
d. Sign the informed consent and agree to complete follow-up.

5) Exclusion criteria:

a. Age > 80 years; 
b. Major operation history one week before admission;

c. Acute coronary events occurred within 3 months before and after stroke;

d. Gastrointestinal hemorrhage or other serious bleeding diseases within one month;

e. Complications including coagulation disorders, mental disorders, pregnant women;

f. Other situations not suitable to participate in the experiment.

6) Test procedure:

a. Cautious physical examination, record body parameters;

b. Paste the fluorescent markers (Figure 1A) and sEMG sensors (Figure 1C), correct equipment;

c. Walk 30 meters on the test platform without exoskeleton;

d. Wear the exoskeleton (Figure 1B), train adaptively and choose appropriate training mode;

e. Walk on the test platform with exoskeleton around 30 minutes (Figure 1E);

f. Test over, questionnaire investigation.

7) Evaluation index:

a. Primary end point: safety of exoskeleton (occurrence of adverse events);

b. Secondary end point: gait function improvement.

8) Possible adverse events:

a. Accidental fall, fracture and other injuries;

b. Other injuries caused by exoskeleton.

\subsection{Exoskeleton design}

Benefiting from the intensive study on mechanical structures, materials, motors and software algorithm, the lower extremity exoskeleton robot designed by our team is lightweight and comfortable, whose mass is $2.6 \mathrm{~kg}$ and is lower than all similar exoskeleton robots on the market ${ }^{[15,25]}$.

According to ergonomics and mechanics, we designed the mechanical structure of the exoskeleton robot (Figure 1B), which meets the requirements of convenient wearing, comfortable carrying and wide adjustable range. At the same time, we adopted carbon fiber as the structure framework, endowing it with characteristics of high strength, low density and low friction resistance ${ }^{[26]}$. Proper motor can reduce the mass of the exoskeleton robot and ensure the user's comfort. By comparing and testing the commonlyused motors, we selected the brushless servo planetary reducer motor (Brand: INNFOS, Model: QDDNU80-6), with rated torque of $6.6 \mathrm{~nm}$, peak torque of $19.8 \mathrm{~nm}$ and weight of only $453.1 \mathrm{~g}$. 
Software control is the other key point to improve the curative effect. The training mode of exoskeleton robot is mainly divided into two kinds: passive and active. The former is relatively mature and has been

put into practice, while the latter is still under research and is considered to be the future direction ${ }^{\text {[27] }}$. The exoskeleton robot used in this clinical study has both passive and active training modes. The training mainly uses the more mature passive mode, while also allows patients to experience the active mode and compare the two modes subjectively.

\subsection{Biomechanical and statistical analysis}

Gait parameters, such as hip angles and toe-ground clearance, were computed by VICON (Nexus 1.8.5, UK, Figure 1E). Plantar pressure was collected and analyzed by Footscan (Footscan 7 gait $2^{\text {nd }}$ generation, Belgium, Figure 1D). Surface electromyogram was acquired through Telemyo 2400R G2 Wireless system (Noraxon, USA, Figure 1C) and the Noraxon MR3.10 software was used for signal processing. MATLAB version R2019a (MathWorks, USA) was used to further process the gait parameters and sEMG data. SPSS 25 was used for Statistic operation. For each condition and result measurement, means and standard deviations were calculated for the paretic and non-paretic limbs. Paired t-test was used to evaluate a small number of comparisons and the statistical significance was set at $a<0.05$.

\section{Results}

\subsection{Participant information}

This study included 9 patients who were hospitalized in the Department of Neurology of Renji Hospital, Shanghai Jiaotong University School of Medicine from January 2018 to December 2019 due to anterior circulation ischemic stroke. All participants finished the test in Shanghai Institute of Traumatology and Orthopaedics from August 2019 to December 2019. The basic information is shown in table 1.

Among the selected stroke patients, 55.6\% (5 / 9) were male, $44.4 \%$ (4 / 9) were female, $61.5 \pm 4.1$ years old (mean \pm standard deviation, the same below), $62.6 \pm 9.4 \mathrm{~kg}$ in weight, $1.63 \pm 0.07 \mathrm{~m}$ in height, $0.82 \pm$ $0.04 \mathrm{~m}$ in lower extremity, $107.2 \pm 40.7$ days after stroke, and the muscle strength of the paretic lower extremity was between 3 and 5 -

\subsection{Gait}

\subsubsection{Hip joint angle}

Drag step is a special phenomenon of hemiplegic patients, which is closely related to the limited hip joint angle ${ }^{[28]}$. Fig.2 B1 illustrates the hip joint angle, and Fig.2 C1 shows the normal hip joint angle curve across the gait cycle. Fig. $2 \mathrm{~A} 1$ shows the hip joint angle curve across the gait cycle of 9 participants in 
turn. In the figure, the black curves represent the conditions without exoskeleton, the colorful curves represent them with exoskeleton, and the shadows represent the variety range of hip joint angle under multiple gait cycles.

The abnormal hip joint angle curve of post-stroke hemiplegic patients is characterized by a decreased amplitude or an unsmooth appearance ${ }^{[29]}$. As Fig. 2 A1 (black) indicates, patients suffer from a limited hip joint movement, leading to drag step. However, with exoskeleton robot assistance (color), most of the subjects (P2, P3, P4, P6, P8, P9) had smoother hip joint angle curves and increased amplitude compared with that without assistance (black).

The maximum hip joint angle was calculated in order to test exoskeleton's effect on patients in a more compelling way. The results show that the average maximum hip joint angle increment after exoskeleton robot assistance reaches $11.3^{\circ}$ on the non-paretic side (Fig.2 D1) and $14.1^{\circ}$ on the paretic side (Fig. 2 E1), which demonstrates that the exoskeleton can increase hip joint angle on both sides of post-stroke hemiplegic patients, and the increment on the paretic side is higher.

\subsubsection{Toe-ground clearance}

Drop foot is another special phenomenon in hemiplegic patients, which is bound up with the limited toeground clearance. The toe-ground clearance is a wildly-used index to measure the severity of drop foot, and can also be used to evaluate the advantage of exoskeleton robot-assisted walking training ${ }^{[30]}$. Fig.2 B2 depicts the toe-ground clearance, and Fig. 2 C2 illustrates the normal toe-ground clearance curve across the gait cycle. Fig. 2 A2 shows the toe-ground clearance curve across the gait cycle of 9 participants in turn. In the figure, the black curves represent circumstances without exoskeleton, the colorful curves represent them with exoskeleton, and the shadows represent the variable range of hip joint angle under multiple gait cycles.

The abnormal toe-ground clearance curve of post-stroke hemiplegic patients is shown with a decreased amplitude, a unsmooth curve and multiple wave peaks ${ }^{[31]}$. As Fig.2 A2 (black) indicates, patients experience limited toe-ground clearance, leading to drop foot. Most of the subjects (P2, P3, P5-P9) had smooth hip joint angle curves and increased amplitude when assisted by exoskeleton robot (color) compared with that without assistance (black).

The maximum toe-ground clearance was calculated in order to further demonstrate the advantage of exoskeleton assistance. The results show that the average maximum toe-ground clearance increment with exoskeleton robot assistance is $63.7 \mathrm{~mm}$ on the non-paretic side (Fig.2 D2) and $73.1 \mathrm{~mm}$ on the paretic side (Fig.2 E2). It indicates that the exoskeleton can increase the toe-ground clearance of poststroke hemiplegic patients on both sides, and the increment on the paretic side is higher.

\subsubsection{Gait symmetry}


Hemiplegic gait manifests a lack of symmetry between the paretic side and the non-paretic side ${ }^{[32]}$. Evaluating and improving the gait symmetry of post-stroke patients helps correct their hemiplegic gait.

The spatial and temporal gait parameters of stroke patients is shown in table 2.

- For the paretic limbs, the cadence, walking speed, stride length, step length decline, and the stride time, single support ascend were compared between assisted and unassisted situation.

- For the non-paretic limbs, the cadence, walking speed decline, and the stride time, step time, single support ascend were compared between assisted and unassisted situation.

The results show that the paretic side holds a slow swing frequency, a large amplitude, and a long support time. The non-paretic side, however, displays a fast swing frequency, a small amplitude, and a short support time. The phenomenon is consistent with the drag step of post-stroke hemiplegic gait ${ }^{[33,34]}$. The results are also related to the setting of training model. In order to ensure the safety of training, the pace is controlled so that some parameters such as walking speed maintained low with exoskeleton assistance.

Radar map is a two-dimensional graph used to demonstrate multiple variables, being able to display regular polygons makes it suitable for symmetry comparison ${ }^{[35]}$. The ratios of the non-paretic side data to the paretic side data were taken as variables to make a radar map. Bilateral symmetry is indicated if the ratio is close to 1 , and the shape of the radar image should be close to a regular polygon. Fig. 3 compares the radar map of gait symmetry with or without exoskeleton assistance. It can be seen that the black radar chart (without the assistance of exoskeleton) is an irregular polygon, and the grey radar chart (with the assistance of exoskeleton) is close to regular polygon. The result indicates that the exoskeleton can improve the gait symmetry of post-stroke hemiplegic patients.

[Please see the supplementary files section to view the equation.]

Q1区

According to the formula (1), we calculated exoskeleton's improvement ratio of gait symmetry. In the formula (1), XN represents non-paretic side parameters without exoskeleton assistance, XP represents paretic side parameters without exoskeleton assistance, $\mathrm{YN}$ represents non-paretic side parameters with exoskeleton assistance, $\mathrm{YN}$ represents paretic side parameters with exoskeleton assistance. The results show that the gait symmetry improved $74.18 \%$ by exoskeleton.

\subsection{Plantar pressure}

Plantar pressure is an effective and burgeoning research methods in the rehabilitation field, which can help assess the compression of different foot area, thus guiding interventions to prevent injuries caused by long-term compression ${ }^{[36]}$. Comparing plantar pressure heat map of P1 (Left hemiplegia, Fig. 4A-B), it can be found that without assistance, the pressure focuses on the healthy side of feet (Fig. 4A), while the pressure distribution tends to be symmetric with exoskeleton assistance (Fig. 4B). Fig. 4C shows ten 
plantar regions according to anatomy in order to better compare the bilateral plantar pressure. It can be found in Fig. 4D that the intensity of pressure in bilateral plantar regions varies a lot with or without assistance of exoskeleton. On the paretic side, the intensity of pressure on Toe 1, Toe 2-5 and Meta 1 increases with exoskeleton assistance. At the same time, the non-paretic side intensity of pressure on Meta 1-3, mid foot and heel medial decreases significantly when assisted by exoskeleton. The results prove that the exoskeleton can decrease the plantar pressure of special regions on the paretic side and increase the plantar pressure of special regions on the non-paretic side, which helps balance the bilateral plantar pressure. We calculated the exoskeleton's improvement ratio of plantar pressure symmetry in the same way by formula (1), and it shows an improvement of $50.82 \%$.

Plantar impulse is the multiplication of pressure and time. As the direct estimate of fatigue accumulation, it is of great significance in evaluating vulnerable parts of the foot ${ }^{[37]}$. Fig. $4 \mathrm{E}$ compares the bilateral plantar impulses in different regions with or without exoskeleton assistance. It is found that when unassisted, the impulse in forefoot accounts for about $70 \%$ of the whole, and the impulse from the nonparetic side is higher than that on the paretic side, indicating that the forefoot is prone to fatigue and damage. Exoskeleton robot helps reduce the impulse of bilateral forefoot, especially that of the nonparetic side, which balances the plantar impulse. We calculated the exoskeleton's improvement ratio of plantar impulse symmetry in the same way by formula (1), and it shows an improvement of $73.79 \%$.

\section{4 sEMG}

The surface electromyogram (sEMG) signal is a non-invasive electro-neurophysiology tool which has been widely used in rehabilitation field for evaluating muscle function ${ }^{[38]}$. sEMG data were rectified and bandpass-filtered $(15$ to $380 \mathrm{~Hz})$ to generate linear envelopes (Fig. 5A ${ }^{[39]}$. The area under the sEMG envelopes were calculated by numerical integration, which is called integral electromyogram (IEMG). IEMG is positively related with muscle strength and activity, which is a practical index for muscle function assessment ${ }^{[19,40]}$. Fig. 5 B compares the IEMG of measured bilateral muscles between circumstances with or without exoskeleton assistance. The IEMG of paretic side in Rectus Femoris, Biceps Femoris Long Head and Tibialis Anterior only equal to $63.90 \% \varangle 57.91 \%$ and $76.77 \%$ of the non-paretic side muscles when unassisted. While the numerical value increase to $98.39 \% \varangle 82.12 \%$ and $83.72 \%$ in turn when assisted by exoskeleton. The results prove that these muscle activities are significantly enhanced by exoskeleton in participants.

\section{Discussion}

Exoskeleton robot is a wearable device combining mechanical equipment and artificial intelligence, which can provide controllable and multi-sensory stimulation, enhance the plasticity of neural connection in the process of movement, and promote the biological and epigenetic changes of nervous system ${ }^{[41]}$. The exoskeleton robot used in this experiment has characteristics of convenient wearing, comfortable structure, high-intensity and low-weight, which is lighter than all listed exoskeletons. It was equipped with 
passive and active auxiliary training modes; the former is relatively mature, and the latter is the future direction of development. In this experiment, three-dimensional gait parameters, plantar pressure and sEMG signals were used to objectively and comprehensively evaluate the improvement of motor function by exoskeleton robot.

Post-stroke hemiplegic patients have drag step, drop foot and asymmetric gait ${ }^{[29,42]}$. Evaluating and correcting the gait parameters, such as improving hip joint angle, toe-ground clearance and gait symmetry, help improve hemiplegic gait, thus promote rehabilitation ${ }^{[43-45]}$. The phenomenon of hemiplegic gait results from post-stoke nervous system damage, which will seriously affect patients' daily life ${ }^{[46]}$. Compared with normal people, post-stroke hemiplegic patients have the characteristics of asymmetric gait, low walking efficiency and higher risk of falling, which are caused by decreased muscle strength, abnormal gait and sensory disorders ${ }^{[47-49]}$. They need to be corrected as soon as possible after stroke for better rehabilitation prognosis ${ }^{[50]}$. Post-stroke hemiplegic patients also have imbalanced weight-bearing on bilateral foot. By evaluating the plantar pressure and correcting them can exoskeleton probably improve patients' walking efficiency and reduce their falling risk ${ }^{[51]}$. The asymmetric plantar pressure and impulse of post-stroke hemiplegic patients can be significantly corrected when assisted by exoskeleton robot. As a non-invasive electro-neurophysiological analysis method, sEMG is widely used in rehabilitation field. IEMG can reflect the intensity of EMG activity, which is a good index for examining and evaluating muscle functions. This study found that the IEMG of rectus femoris, biceps femoris longhead and tibialis anterior muscle in the paretic side was significantly lower than that in the nonparetic side. While the exoskeleton can significantly improve the IEMG of these muscles, it means that the exoskeleton positively stimulates these muscles to generate activities and helps restore motor functions ${ }^{[52]}$.

In the nervous system, the diseases of central nerve, peripheral nerve, neuromuscular junction and muscle can lead to lower extremity dyskinesia ${ }^{[53]}$. There are many other clinical studies on exoskeleton in patients with lower extremity motor dysfunction, such as spinal cord injury ${ }^{[54]}$, postoperative ${ }^{[55]}$, agerelated gait disorder ${ }^{[14]}$, child cerebral palsy ${ }^{[39]}$, multiple sclerosis ${ }^{[56]}$ and Parkinson's disease ${ }^{[57]}$, etc. Our research findings not only exhibit more accurate clinical requirements for the research and renovation of exoskeleton robot, but also provides a new vision for clinical motor function improvement of lower extremity, including in stroke patients. When developing exoskeleton robot, we need to emphasize gait characteristics of different diseases, such as hemiplegia of stroke and paraplegia of spinal cord injury, so as to better design the hardware structure and software algorithm.

There are many factors that affect the rehabilitation effect of exoskeleton, including active or passive training mode, training frequency and intensity, different severity and course stages of stroke ${ }^{[58]}$. The training intensity, frequency and start time have not formed a unified standard. Some studies have pointed out that increasing training intensity, increasing training times, and task-based training can promote neural plasticity and functional recovery. The most effective rehabilitation interventions, including gait training, must be conducted in an intensive and task-oriented manner shortly after stroke, 
and should include multi-sensor stimulation ${ }^{[59]}$. In this study, we used a small sample and selfcomparison methods to do exploratory research because our exoskeleton is newly developed. In the future, we should further explore the rehabilitating function of exoskeleton robot in stroke hemiplegia patients, as well as in other motor dysfunction patients. In the future, we should continue to explore the mechanisms of exoskeleton robot on the rehabilitation of patients with lower extremity movement disorders, so as to provide a better theoretical basis for its clinical application.

\section{Conclusion}

This study proves that, first, our exoskeleton robot can correct the asymmetry of gait, add to the distance between toes and ground, and increase the angle of hip joint in post-stroke hemiplegic patients. Second, it can improve the symmetry of bilateral plantar pressure, alleviate the pressure of the healthy plantar and reduce the impulse of bilateral forefeet, which might help avoid plantar injuries caused by long-term abnormal gaits. Third, it can also enhance the integral EMG of Rectus Femoris, Biceps Femoris Long Head and Tibialis Anterior, which implies its positive effect on stimulating corresponding muscles to produce activities. Moreover, this study provides important data for the research and renovation of exoskeleton robot, verifies the significance of exoskeleton robot in rehabilitating stroke patients, and offers a new perspective for assisted-walking as well as rehabilitation in other motor dysfunction-related diseases.

\section{Declarations}

\subsection{Ethics approval and consent to participate}

This clinical research was approved by Ruijin Hospital Ethics Committee, Shanghai Jiaotong University School of Medicine. All participants had signed the informed consent.

\subsection{Consent for publication}

All presentations of this article have consent for publication

\subsection{Availability of data and material}

The datasets analysed during the current study available from the corresponding author on reasonable request.

\subsection{Competing interests}

The authors declare no competing interest. 


\subsection{Funding}

This work was funded by Innovative research team of high-level local universities in Shanghai, National Natural Science Foundation of China $(8147219,81771295)$, Special Foundation for Integration of Traditional Chinese Medicine and Western Medicine (ZHYY-ZXJHZX-1-201701), Health and Family Planning Committee Foundation of Pudong New Area, Shanghai (PW2018D-02).

\subsection{Authors' contributions}

K.W, Hj.Y and Hm.Z contributed equally to this work. K.W and P.H conducted the experiments. K.W, Hm.Z and P.H analyzed the data. K.W and Hm.Z prepared the figures and tables. K.W, Hj.Y and J.P prepared the manuscript. Wx.Y provides the exoskeleton robot. K.W and Yt.G conceptualized the project. Yt.G P.H and Wx.Y supervised the project. All coauthors have reviewed and approve the contents of the manuscript.

\subsection{Acknowledgement}

We would like to acknowledge Prof. Weixin Yan and his team for offering the exoskeleton robot and Researcher Ping Huang for providing a great laboratory platform for this project.

\section{Tables}

Table 1. Participant information. 


\begin{tabular}{|c|c|c|c|c|c|c|c|}
\hline No. & Gender & $\begin{array}{c}\text { Age } \\
\text { (years) }\end{array}$ & $\begin{array}{c}\text { Weight } \\
(\mathrm{kg})\end{array}$ & $\begin{array}{l}\text { Height } \\
\text { (meter) }\end{array}$ & $\begin{array}{c}\text { Leg } \\
\text { (meter) }\end{array}$ & $\begin{array}{l}\text { Post-stroke } \\
\text { (days) }\end{array}$ & $\begin{array}{c}\text { Affected side } \\
\text { Muscle strength }\end{array}$ \\
\hline 1 & Male & 59 & 71 & 1.70 & 0.85 & 79 & Left 5- \\
\hline 2 & Male & 56 & 74 & 1.75 & 0.88 & 52 & Right 4 \\
\hline 3 & Female & 64 & 75 & 1.64 & 0.87 & 119 & Right 4+ \\
\hline 4 & Male & 59 & 61 & 1.65 & 0.83 & 110 & Left 3 \\
\hline 5 & Male & 59 & 52 & 1.54 & 0.78 & 139 & Right 4+ \\
\hline 6 & Female & 61 & 62 & 1.60 & 0.77 & 49 & Right 4 \\
\hline 7 & Female & 61 & 63 & 1.62 & 0.82 & 129 & Left 3 \\
\hline 8 & Female & 69 & 57 & 1.55 & 0.78 & 173 & Left 4- \\
\hline 9 & Male & 66 & 48 & 1.59 & 0.80 & 115 & Left 4- \\
\hline
\end{tabular}

Table 2 Spatial and temporal gait parameters of stroke patients 


\section{Unassisted Exo-assisted Unassisted Exo-assisted}

\begin{tabular}{ccccc} 
Cadence(steps/min) & $81.88 \pm$ & $53.52 \pm$ & $88.56 \pm 23.45$ & $52.42 \pm 21.98^{* *}$ \\
& 29.12 & $22.21^{*}$ & & \\
Walking Speed(m/s) & $0.60 \pm 0.25$ & $0.32 \pm 0.21^{*}$ & $0.61 \pm 0.24$ & $0.32 \pm 0.22^{*}$ \\
Stride Time(s) & $1.71 \pm 0.70$ & $2.55 \pm 0.79^{*}$ & $1.48 \pm 0.49$ & $2.61 \pm 0.83^{* *}$ \\
Step Time(s) & $1.00 \pm 0.58$ & $1.41 \pm 0.54$ & $0.69 \pm 0.22$ & $1.23 \pm 0.30^{* *}$ \\
Opposite Foot Off(\%) & $19.76 \pm 9.50$ & $16.82 \pm 8.79$ & $22.20 \pm 9.74$ & $17.08 \pm 8.10$ \\
Opposite Foot & $48.51 \pm 6.17$ & $48.99 \pm 7.34$ & $52.35 \pm 7.61$ & $53.03 \pm 8.43$ \\
Contact(\%) & & & & \\
Foot Off(\%) & $54.76 \pm 8.26$ & $62.77 \pm 8.31$ & $58.46 \pm 8.77$ & $63.41 \pm 8.70$ \\
Single Support(\%) & $0.59 \pm 0.15$ & $0.79 \pm 0.17 *$ & $0.64 \pm 0.20$ & $0.90 \pm 0.30^{*}$ \\
Double Support(\%) & $0.44 \pm 0.29$ & $0.81 \pm 1.20$ & $0.40 \pm 0.24$ & $0.75 \pm 0.47$ \\
Stride Length(m) & $0.89 \pm 0.21$ & $0.68 \pm 0.18^{*}$ & $0.83 \pm 0.18$ & $0.68 \pm 0.20$ \\
Step Length(m) & $0.47 \pm 0.16$ & $0.36 \pm 0.09^{*}$ & $0.45 \pm 0.15$ & $0.35 \pm 0.11$ \\
\hline
\end{tabular}

Values are presented as mean \pm standard deviation; $* \mathrm{P}<0.05$ and $* * \mathrm{P}<0.01$ vs. Unassisted.

\section{References}

[1] COLLABORATORS G B D S. Global, regional, and national burden of stroke, 1990-2016: a systematic analysis for the Global Burden of Disease Study 2016 [J]. Lancet Neurol, 2019, 18(5): 439-58.

[2] WU S, CHENG Y, WU B, et al. Stroke research in 2019: towards optimising treatment and prevention [J]. Lancet Neurol, 2020, 19(1): 2-3.

[3] LESLI, SKOLARUS, WILLIAM, et al. Marked Regional Variation in Acute Stroke Treatment Among Medicare Beneficiaries [J]. Stroke A Journal of Cerebral Circulation, 2015,

[4] STINEAR C M, LANG C E, ZEILER S, et al. Advances and challenges in stroke rehabilitation [J]. Lancet Neurol, 2020, 
[5] ORTLIEB A, BOURI M, BAUD R, et al. An assistive lower limb exoskeleton for people with neurological gait disorders [J]. IEEE Int Conf Rehabil Robot, 2017, 2017(441-6.

[6] MCGLINCHEY M P, JAMES J, MCKEVITT C, et al. The effect of rehabilitation interventions on physical function and immobility-related complications in severe stroke: a systematic review [J]. BMJ Open, 2020, 10(2): e033642.

[7] CHEN G, CHAN C K, GUO Z, et al. A review of lower extremity assistive robotic exoskeletons in rehabilitation therapy [J]. Crit Rev Biomed Eng, 2013, 41(4-5): 343-63.

[8] CHEN B, ZHONG C H, ZHAO X, et al. A wearable exoskeleton suit for motion assistance to paralysed patients [J]. J Orthop Translat, 2017, 11(7-18.

[9] SANCHEZ-VILLAMANAN M D C, GONZALEZ-VARGAS J, TORRICELLI D, et al. Compliant lower limb exoskeletons: a comprehensive review on mechanical design principles [J]. J Neuroeng Rehabil, 2019, 16(1): 55.

[10] BORTOLE M, VENKATAKRISHNAN A, ZHU F, et al. The H2 robotic exoskeleton for gait rehabilitation after stroke: early findings from a clinical study [J]. J Neuroeng Rehabil, 2015, 12(54.

[11] WINSTEIN C J, WOLF S L, DROMERICK A W, et al. Effect of a Task-Oriented Rehabilitation Program on Upper Extremity Recovery Following Motor Stroke: The ICARE Randomized Clinical Trial [J]. JAMA, 2016, 315(6): 571-81.

[12] YTTERBERG C, KRISTENSEN H K, TISTAD M, et al. Factors related to met needs for rehabilitation 6 years after stroke [J]. PLoS One, 2020, 15(1): e0227867.

[13] TAKETOMI M, SHIMIZU Y, KADONE H, et al. Hybrid Assistive Limb Intervention in a Patient with Late Neurological Deterioration after Thoracic Myelopathy Surgery due to Ossification of the Ligamentum Flavum [J]. Case Rep Orthop, 2018, 2018(6171760.

[14] LEE S H, LEE H J, CHANG W H, et al. Gait performance and foot pressure distribution during wearable robot-assisted gait in elderly adults [J]. J Neuroeng Rehabil, 2017, 14(1): 123.

[15] SEO K, LEE J, PARK Y J. Autonomous hip exoskeleton saves metabolic cost of walking uphill [J]. IEEE Int Conf Rehabil Robot, 2017, 2017(246-51.

[16] YAN H, YANG C J. Design and validation of a lower limb exoskeleton employing the recumbent cycling modality for post-stroke rehabilitation [J]. P I Mech Eng C-J Mec, 2014, 228(18): 3517-25.

[17] HORTAL E, UBEDA A, IANEZ E, et al. EEG-Based Detection of Starting and Stopping During Gait Cycle [J]. Int J Neural Syst, 2016, 26(7): 1650029. 
[18] RODRIGUEZ-UGARTE M, IANEZ E, ORTIZ M, et al. Improving Real-Time Lower Limb Motor Imagery Detection Using tDCS and an Exoskeleton [J]. Front Neurosci, 2018, 12 (757.

[19] ANDROWIS G J, PILKAR R, RAMANUJAM A, et al. Electromyography Assessment During Gait in a Robotic Exoskeleton for Acute Stroke [J]. Front Neurol, 2018, 9(630.

[20] RAJASEKARAN V, LOPEZ-LARRAZ E, TRINCADO-ALONSO F, et al. Volition-adaptive control for gait training using wearable exoskeleton: preliminary tests with incomplete spinal cord injury individuals [J]. J Neuroeng Rehabil, 2018, 15(1): 4.

[21] SCHWARTZ I, SAJIN A, FISHER I, et al. The Effectiveness of Locomotor Therapy Using RoboticAssisted Gait Training in Subacute Stroke Patients: A Randomized Controlled Trial [J]. Pm \& R the Journal of Injury Function \& Rehabilitation, 2009, 1(6): 516-23.

[22] MOLTENI F, GASPERINI G, GAFFURI M, et al. Wearable robotic exoskeleton for overground gait training in sub-acute and chronic hemiparetic stroke patients: preliminary results [J]. Eur $\mathrm{J}$ Phys Rehabil Med, 2017, 53(5): 676-84.

[23] CALABRO R S, NARO A, RUSSO M, et al. Shaping neuroplasticity by using powered exoskeletons in patients with stroke: a randomized clinical trial [J]. J Neuroeng Rehabil, 2018, 15(1): 35.

[24] CHO J E, YOO J S, KIM K E, et al. Systematic Review of Appropriate Robotic Intervention for Gait Function in Subacute Stroke Patients [J]. Biomed Res Int, 2018, 2018(4085298.

[25] JAYARAMAN A, O'BRIEN M K, MADHAVAN S, et al. Stride management assist exoskeleton vs functional gait training in stroke: A randomized trial [J]. Neurology, 2019, 92(3): e263-e73.

[26] FRISOLI A, ROCCHI F, MARCHESCHI S, et al. A new force-feedback arm exoskeleton for haptic interaction in virtual environments; proceedings of the Eurohaptics Conference, 2005 and Symposium on Haptic Interfaces for Virtual Environment and Teleoperator Systems, 2005 World Haptics 2005 First Joint, $\mathrm{F}, 2005[\mathrm{C}]$.

[27] VLUGGEN T, VAN HAASTREGT J C M, TAN F E S, et al. Factors associated with successful home discharge after inpatient rehabilitation in frail older stroke patients [J]. BMC Geriatr, 2020, 20(1): 25.

[28] AFSCHRIFT M, DE GROOTE F, DE SCHUTTER J, et al. The effect of muscle weakness on the capability gap during gross motor function: a simulation study supporting design criteria for exoskeletons of the lower limb [J]. Biomed Eng Online, 2014, 13(111.

[29] OLNEY S J, RICHARDS C. Hemiparetic gait following stroke. Part l: Characteristics [J]. Gait \& Posture, 4(2): 0-148.

[30] ZHANG C, ZHU Y, FAN J, et al. Design of a quasi-passive 3 DOFs ankle-foot wearable rehabilitation orthosis [J]. Biomed Mater Eng, 2015, 26 Suppl 1(S647-54. 
[31] VACHALOVA I, HECKMAN J G. Differential diagnosis of foot drop: foot drop stroke syndrome [J]. Journal of Surgical Orthopaedic Advances, 2014, 23(2): 122-3.

[32] BONINI-ROCHA A C, DE ANDRADE A L S, MORAES A M, et al. Effectiveness of Circuit-Based Exercises on Gait Speed, Balance, and Functional Mobility in People Affected by Stroke: A Meta-Analysis [J]. PM R, 2018, 10(4): 398-409.

[33] PARK E J, KANG J, SU H, et al. Design and preliminary evaluation of a multi-robotic system with pelvic and hip assistance for pediatric gait rehabilitation [J]. IEEE Int Conf Rehabil Robot, 2017, 2017(3329.

[34] KOOPMAN B, VAN ASSELDONK E H, VAN DER KOOIJ H. Selective control of gait subtasks in robotic gait training: foot clearance support in stroke survivors with a powered exoskeleton [J]. $J$ Neuroeng Rehabil, 2013, 10(3.

[35] LIN L C, CHUANG H S. Analyzing the locomotory gaitprint of Caenorhabditis elegans on the basis of empirical mode decomposition [J]. PLoS One, 2017, 12(7): e0181469.

[36] COCK A D, WILLEMS T, WITVROUW E, et al. A functional foot type classification with cluster analysis based on plantar pressure distribution during jogging [J]. Gait \& Posture, 23(3): 0-347.

[37] HUANG P Y B, DENG LF, ZHU WY, XIA J, HUANG QY. . Gait features and foot plantar pressure distribution of nurse natural walking on flat ground [J]. Zhongguo Zuzhi Gongcheng Yanjiu, 2012, 16(7)): 1259-62.

[38] HU J, HOU Z G, PENG L, et al. sEMG-Based Single-Joint Active Training with iLeg-A Horizontal Exoskeleton for Lower Limb Rehabilitation [J]. Lect Notes Comput Sc, 2014, 8836(535-42.

[39] LERNER Z F, DAMIANO D L, BULEA T C. A lower-extremity exoskeleton improves knee extension in children with crouch gait from cerebral palsy [J]. Sci Transl Med, 2017, 9(404):

[40] WANG W, LI K, YUE S, et al. Associations between lower-limb muscle activation and knee flexion in post-stroke individuals: A study on the stance-to-swing phases of gait [J]. Plos One, 2017, 12(9): e0183865.

[41] MOLTENI F, GASPERINI G, CANNAVIELLO G, et al. Exoskeleton and End-Effector Robots for Upper and Lower Limbs Rehabilitation: Narrative Review [J]. PM R, 2018, 10(9S2): S174-S88.

[42] VISINTIN M. A new approach of retain gait in stroke patients through body weight support and treadmill stimulation [J]. Stroke, 1998, 29(

[43] PATTERSON K K, GAGE W H, BROOKS D, et al. Evaluation of gait symmetry after stroke: A comparison of current methods and recommendations for standardization [J]. 31(2): 0-246. 
[44] BRODERICK P, HORGAN F, BLAKE C, et al. Mirror therapy for improving lower limb motor function and mobility after stroke: A systematic review and meta-analysis [J]. Gait \& Posture, 2018, 63(

[45] TISSERAND R, ARMAND S, ALLALI G, et al. Cognitive-motor dual-task interference modulates mediolateral dynamic stability during gait in post-stroke individuals [J]. Human Movement Science, 2018, 58(175-84.

[46] DUNNING K, O'DELL M W, KLUDING P, et al. Peroneal Stimulation for Foot Drop After Stroke: A Systematic Review [J]. American Journal of Physical Medicine \& Rehabilitation, 2015, 94(8): 649.

[47] NUNZIO A M D, ZUCCHELLA C, SPICCIATO F, et al. Biofeedback rehabilitation of posture and weightbearing distribution in stroke: A center of foot pressure analysis [J]. Functional Neurology, 2014, 29(2): 1-8.

[48] YANG D J, PARK S K, KIM J H, et al. Effect of changes in postural alignment on foot pressure and walking ability of stroke patients [J]. Journal of Physical Therapy Science, 27(9): 2943-5.

[49] YANG S R, HEO S Y, LEE H J. Immediate effects of kinesio taping on fixed postural alignment and foot balance in stroke patients [J]. Journal of Physical Therapy Science, 27(11): 3537-40.

[50] JAKUBOWITZ E, YAO D, WINDHAGEN H, et al. [Treatment Options for Neurogenic Drop Foot: A Systematic Literature Research] [J]. 2017,

[51] BULDT A K, FORGHANY S, LANDORF K B, et al. Foot posture is associated with plantar pressure during gait: A comparison of normal, planus and cavus feet

[J]. Gait \& Posture, 62(235-40.

[52] ROY S H, CHENG M S, CHANG S S, et al. A Combined sEMG and Accelerometer System for Monitoring Functional Activity in Stroke [J]. IEEE Transactions on Neural Systems \& Rehabilitation Engineering, 2010, 17(6): 585-94.

[53] YOUNG A J, FERRIS D P. State of the Art and Future Directions for Lower Limb Robotic Exoskeletons [J]. IEEE Trans Neural Syst Rehabil Eng, 2017, 25(2): 171-82.

[54] CHEUNG E Y Y, NG T K W, YU K K K, et al. Robot-Assisted Training for People With Spinal Cord Injury: A Meta-Analysis [J]. Arch Phys Med Rehabil, 2017, 98(11): 2320-31 e12.

[55] PUENTES S, KADONE H, KUBOTA S, et al. Reshaping of Gait Coordination by Robotic Intervention in Myelopathy Patients After Surgery [J]. Front Neurosci, 2018, $12(99$.

[56] STRAUDI S, BENEDETTI M G, VENTURINI E, et al. Does robot-assisted gait training ameliorate gait abnormalities in multiple sclerosis? A pilot randomized-control trial [J]. NeuroRehabilitation, 2013, 33(4): 555-63. 
[57] KANG M G, YUN S J, SHIN H I, et al. Effects of robot-assisted gait training in patients with Parkinson's disease: study protocol for a randomized controlled trial [J]. Trials, 2019, 20(1): 15.

[58] DENG W, PAPAVASILEIOU I, QIAO Z, et al. Advances in Automation Technologies for Lower Extremity Neurorehabilitation: A Review and Future Challenges [J]. IEEE Rev Biomed Eng, 2018, 11(289305.

[59] CHANG W H, KIM M S, HUH J P, et al. Effects of robot-assisted gait training on cardiopulmonary fitness in subacute stroke patients: a randomized controlled study [J]. Neurorehabil Neural Repair, 2012, 26(4): 318-24.

\section{Figures}

$\mathbf{A}$
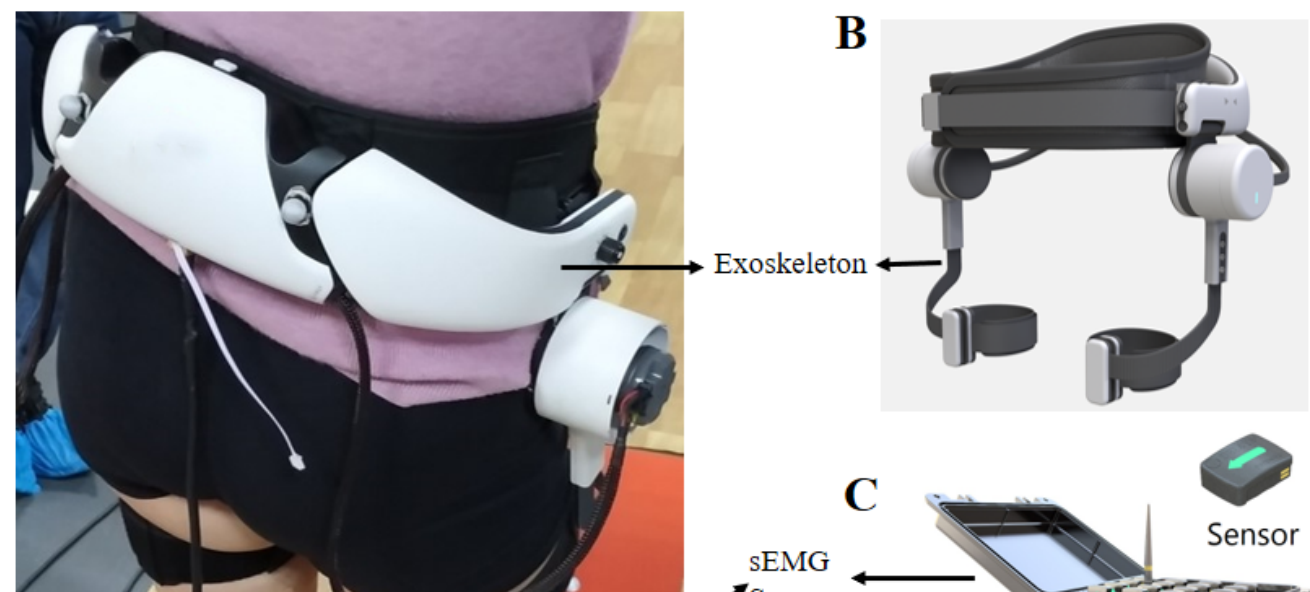

$\mathbf{E}$

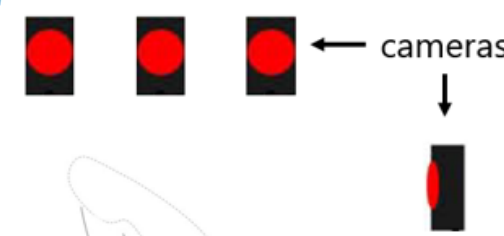

Exoskeleton
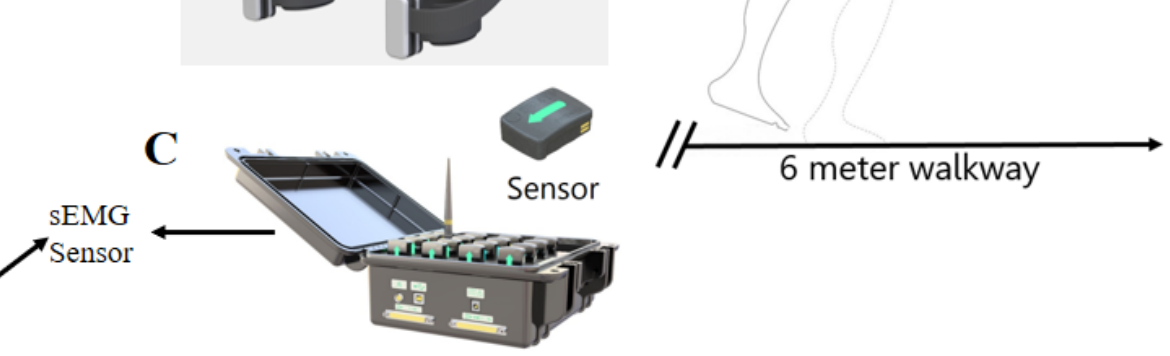

\section{Figure 1}

Lower-extremity exoskeleton robot study description. A) Image illustrating exoskeleton and measuring tools on a study participant; B) Front view of the lower extremity exoskeleton; C) Sensors of Telemyo 2400R G2 Wireless system (Noraxon, USA); D) Footscan pressure plate (RSscan, Belgium); E) Image illustrating participants walk under the monitor of 3D motion capture system (VICON, UK). 

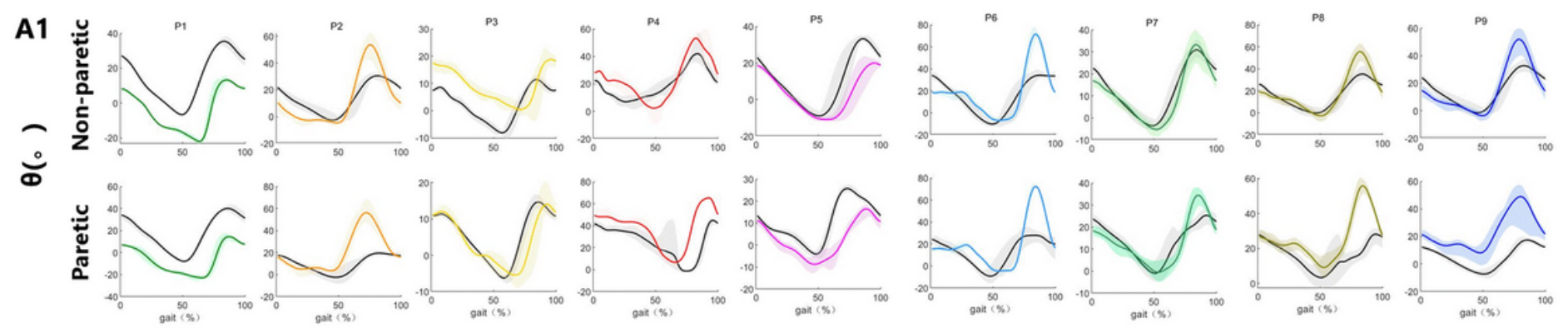

B1

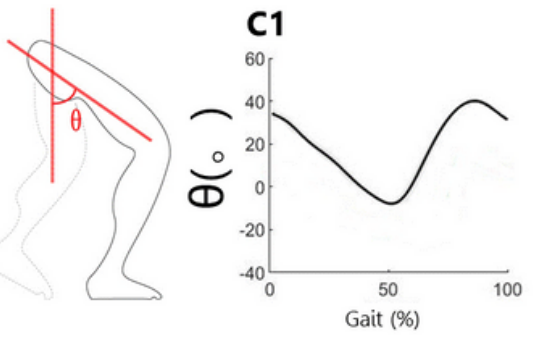

D1

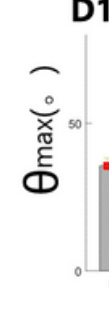

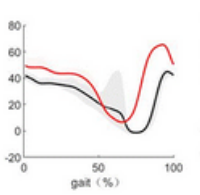

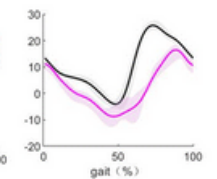

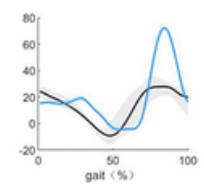

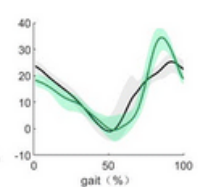

E1

Non-paretic $\Delta_{\text {avg }}=11.3^{\circ} \quad \mathrm{P}=0.098$

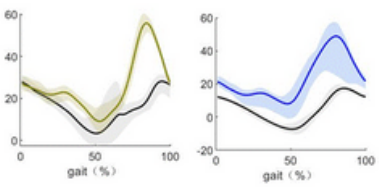

Paretic $\Delta$ avg $=14.1^{\circ} \quad \mathrm{P}=0.102$
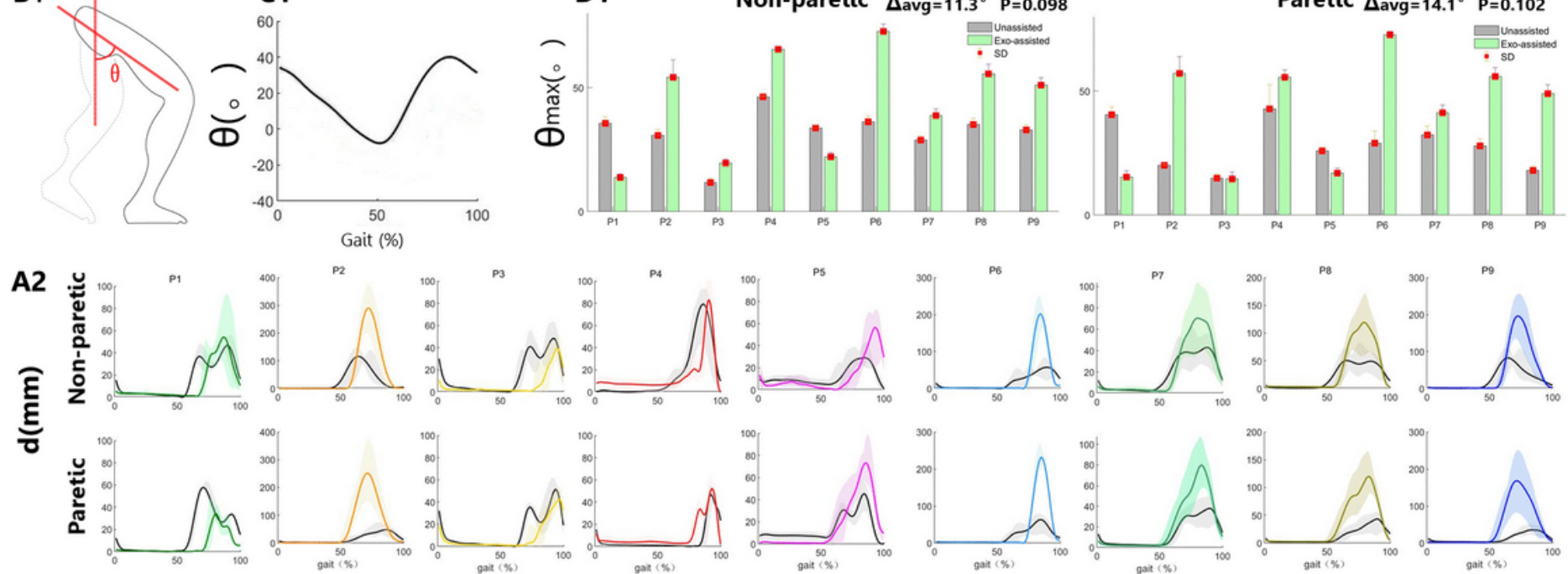

B2

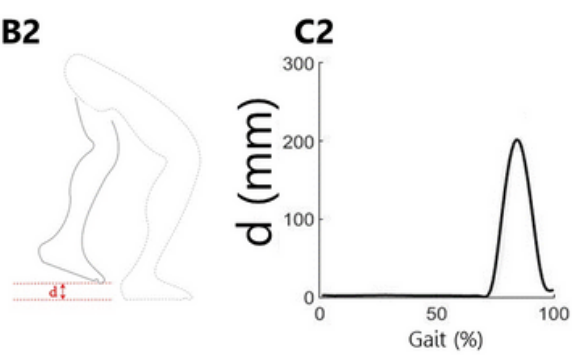

D2

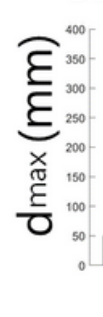

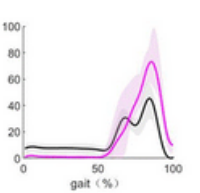

Non-paretic

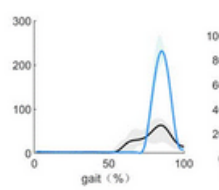

$\Delta$ av

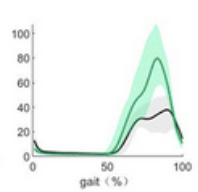

E2

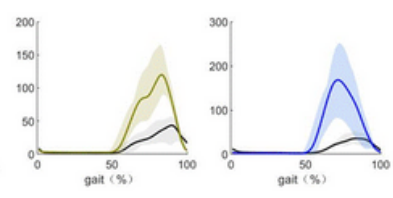

Paretic $_{\Delta \text { avg }=71.6 \mathrm{~mm} \mathrm{P}=0.029}$

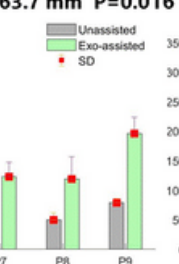
$\square$ Unassisted
Exo-assistod
SD

\section{Figure 2}

Variance of hip angle and Toe-ground clearance. A1) P1-P9: Hip angle across the gait cycle of nine patients, Black lines: non-assisted, Colored lines: exo-assisted, Shade: variance range of hip angle, B1) Sketch Map of hip angle, C1) Variance of toe-ground clearance, D1, E1) Maximum hip angle histogram of Paretic and Non-paretic limbs, $\Delta$ avg: Average of maximum hip angle increment, P: The significance of paired t-test results (maximum hip angle of unassisted and exo-assisted). A2) P1-P9: Toe-ground clearance across the gait cycle of nine patients, Black lines: non-assisted, Colored lines: exo-assisted, Shade: variance range of toe-ground clearance, B2) Sketch Map of Toe-ground clearance, C2) Toe-ground clearance across the gait cycle, D2, E2) Maximum toe-ground clearance histogram of Paretic and Nonparetic limbs, $\Delta$ avg: Average of maximum toe-ground clearance increment, $P$ : The significance of paired ttest results (maximum toe-ground clearance of unassisted and exo-assisted). 


\section{Cadence \\ 1.50}

Step Length Walking Speed

Stride Length

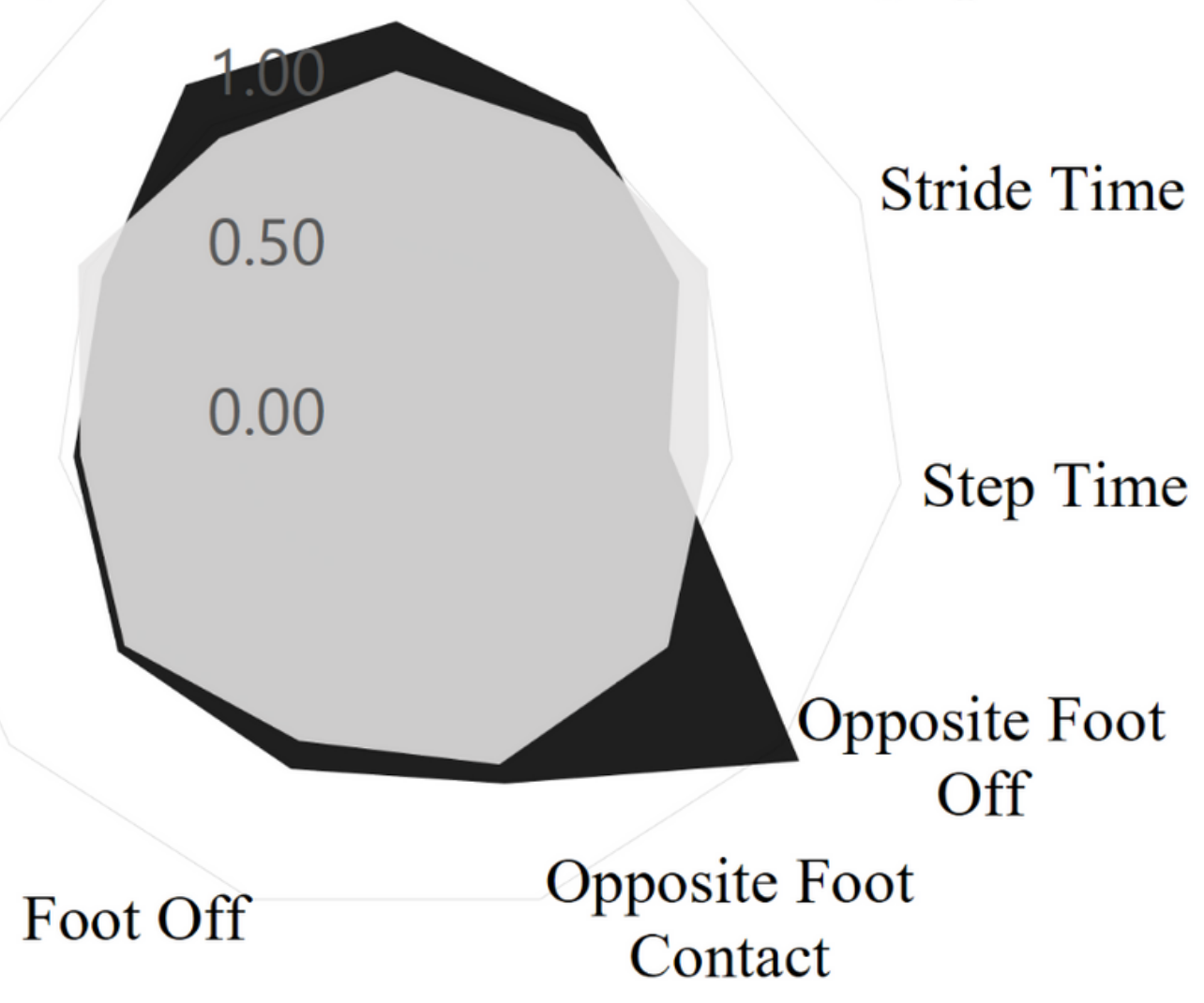

Stride Time

Double Support

Single Support

Figure 3

Radar chart of gait symmetry, Grey Polygon represents radar map with the assistance of exoskeleton and Black Polygon represents radar map without the assistance of exoskeleton. 
A

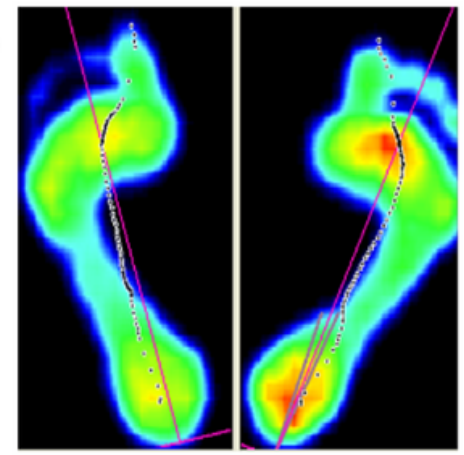

B

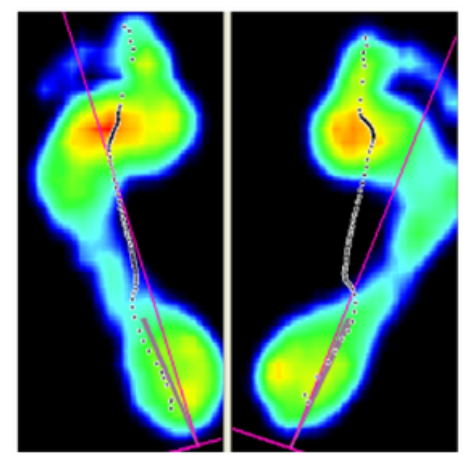

C

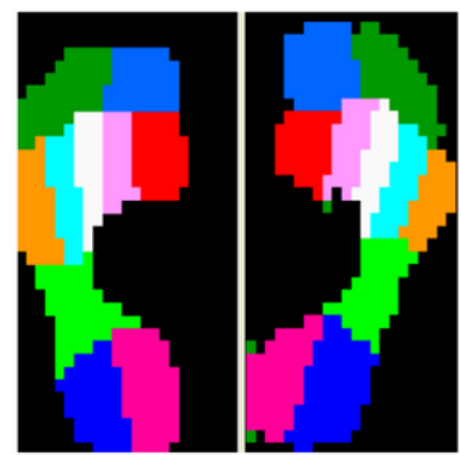

Heel Lateral

Heel Medial

Midfoot

Meta 5

Meta 4

Meta 3

Meta 2

Meta 1

Toe 2-5

Toe1
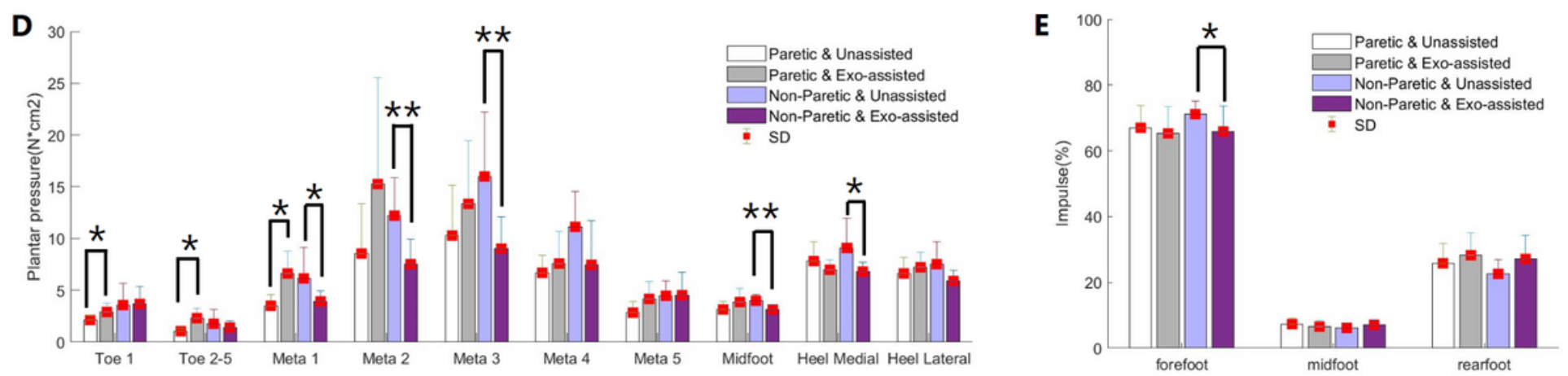

Figure 4

Plantar pressure. A) Plantar pressure heat map of P1 when unassisted, B) Plantar pressure heat map of P1when assisted by exoskeleton, C) plantar regions according to anatomy, D) Intensity of pressure in different plantar divisions, E) Impulse distribution of different plantar divisions, ${ }^{*} P<0.05,{ }^{*} P<0.01$.

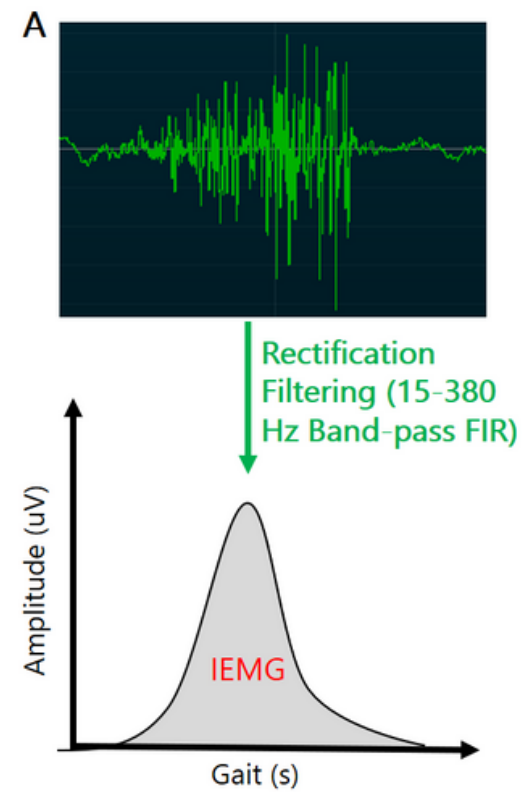

B
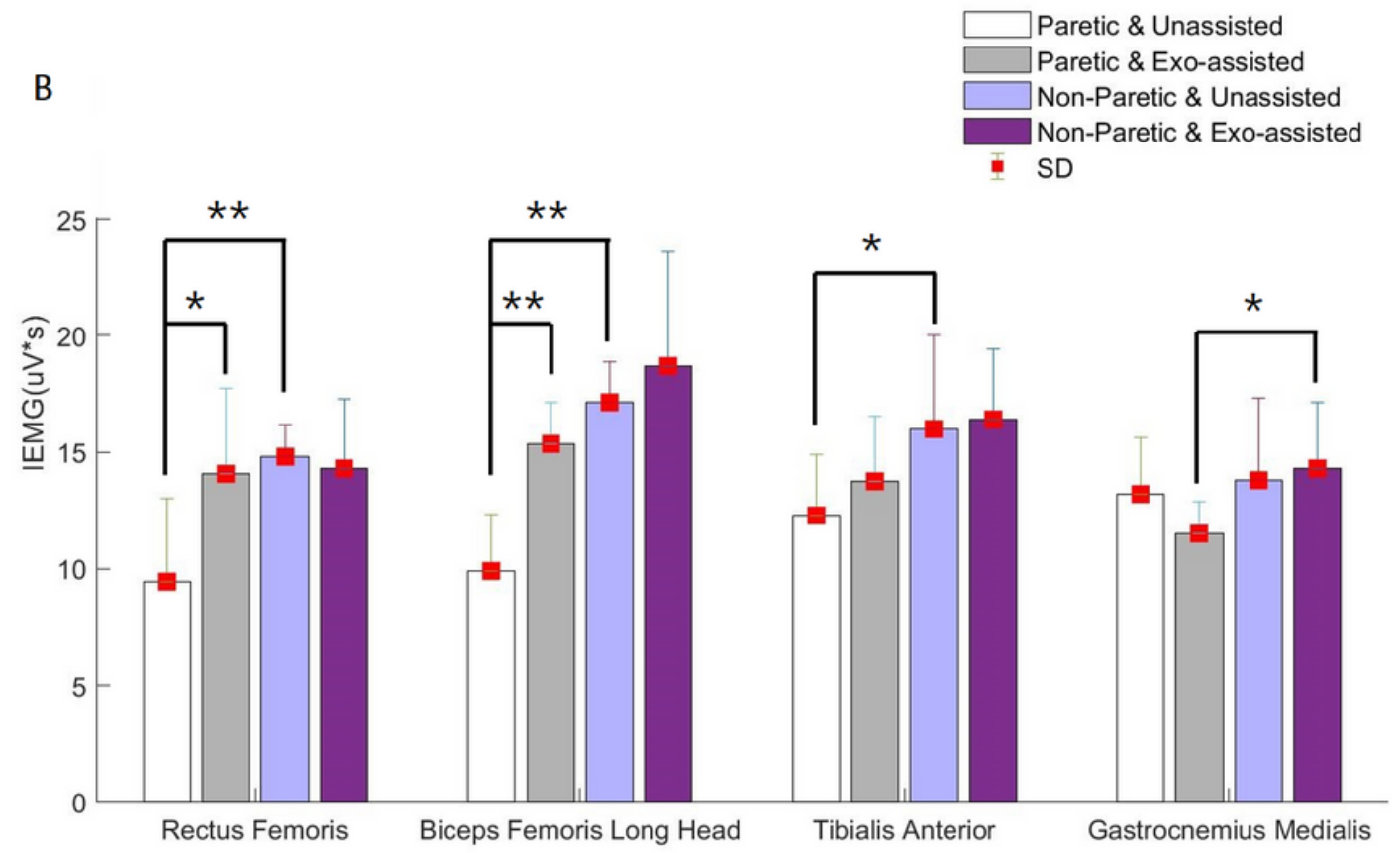

Figure 5 
Surface electromyography. A) Surface electromyography signal processing (IEMG: Integral Electromyogram), B), Histogram of IEMG, * $P<0.05{ }^{\star \star} P<0.01$.

\section{Supplementary Files}

This is a list of supplementary files associated with this preprint. Click to download.

- Equation.docx 